\title{
Refining Parkinson's Neurological Disorder Identification Through Deep Transfer Learning
}

\author{
Amina Naseer, Monail Rani, Saeeda Naz, Muhammad I Razzak, Muhammad Imran, and Guandong Xu
}

\begin{abstract}
Parkinson's disease (PD), a multi-system neurodegenerative disorder which affects the brain slowly, is characterized by symptoms such as muscle stiffness, tremor in the limbs and impaired balance, all of which tend to worsen with the passage of time. Available treatments target its symptoms, aiming to improve the quality of life, however, automatic diagnosis at early stages is still a challenging medicine-related task to date, since a patient may have an identical behavior to that of a healthy individual at the very early stage of the disease. Parkinson's disease detection through handwriting data is a significant classification problem for identification of PD at the infancy stage. In this paper, a PD identification is realized with help of handwriting images that helps as one of the earliest indicators for PD. For this purpose, we proposed a deep convolutional neural network classifier with transfer learning and data augmentation techniques to improve the identification. Two approaches like freeze and fine-tuning of transfer learning are investigated using ImageNet and MNIST dataset as source task independently. A trained network achieved $\mathbf{9 8 . 2 8 \%}$ accuracy using fine-tuning based approach using ImageNet and $\mathrm{PaHaW}$ dataset. Experimental results on benchmark dataset reveal that proposed approach provides better detection of Parkinson's disease as compared to state of the art work.

Keywords: Parkinson disease, handwriting analysis, neurodegenerative disorder
\end{abstract}

\section{Introduction}

Walking into the bedroom, using technology gadgets, washing dishes, reading newspaper, writing or typing text etc. all of these daily based actions involve movement. We never noticed that how brain works, we never think twice about the certain action before performing it, that goes into brain and makes the movement possible, however, some of us suffer

- Amina Naseer, Monail Rani and Saeeda Naz are with Computer Science Department, GGPC.No.1 Abbottabad, Higher Education Department, KPK, Pakistan.

E-mail: saeedanaz292@gmail.com

- Muhammad Imran Razzak and Guandong Xu is with Advanced Analytics Institute, University of Technology, Sydney, Australia, NSW, 2007.

E-mail: imran.razzak@ieee.org this issue i.e. movement disorder may be possible in case ones process go awry due something happens to brains deep parts basal ganglia and the substantia nigra and you lose control over the motor system. Deficiency results in change in speech and movement, depression and anxiety. Parkinsons disease is the best known movement disorder.

Worryingly the global prevalence of Parkinson Disease (PD) is increasing over time. Currently, about 1 million Americans and 10 million people world wide suffer from Parkinson's, a progressive neurological disorder according to APDA (American Parkinson Disease Association) [1]. Famous patientsturned-advocates include Muhammad Ali, Michael J. Fox and Janet Reno. It is expected to double within the next 20 years (up to $2 \%$ and $6 \%$ in people over the age of 60 and 80 years respectively). Currently, the cure for Parkinson disease is not available, and the therapies can only help to control motor symptoms by reversing the DA deficiency [2]. Furthermore, there's no single test for Parkinson's diagnosis, moreover, there is no clear indication of what causes Parkinson, although physician technically understand what happens with the patient. Unlike other disease diagnosis, genetic models even do not generate important cardinal features of PD [2, 3]. However, there are traditional aproaches for diagnosis and investigation of $\mathrm{PD}$ which are invasive methods like Computed tomography (CT) scan, MRI, X-rays, PET, SPECT ("Single Photon Emission Computerized Tomography") / DAT ("Dopamine Transporter") scan, ultrasound, etc which are costly and can be effective when disease is spread over the brain. We require non-invasive and clinical screening test to diagnose PD at an early stage and assist the physician to cure and avoid the spreading of the Parkinson's disease in other cells of the brain.

Recently, several machine based systems have been proposed to identify the early symptoms of Parkinsons and similar neurological diseases and different mode of input has been used such as voice, handwritten, speech patterns to observe subtle. Furthermore, muscular movements devices such as wearable sensors as also been used for PD identification. Among Parkinsons Disease (PD) motor symptoms, freezing of gait 
(FOG) may be the most incapacitating. Aside from the primary motor symptoms, difficulties starts to occur frequently in handwriting of patient i.e. a major complaint of patients with PD is their inability to sign legibly.This phenomena is generally known as micrographia (abnormally small, cramped handwriting or the progression to progressively smaller handwriting) and considered as a biomarker for detection of Parkinson's disease i.e. researches have revealed that about 63 percent of Parkinson's patients have suffered from micrographia [4]. It is the one the most commonly reported and easily detectable handwriting abnormality in patients with PD. However, micrographia is perhaps the tip of the iceberg representing the handwriting abnormalities in PD [5]. Studies have shown that there is a strong evidences of correlation between handwriting changes and problems in the nervous system [6-9]. The symptoms of handwriting impairment in Parkinsons patients are less handwriting speed, low pressure, velocity, less continuity as compared to healthy ones. So handwriting analysis is an efficient approach for detection of disease as compare to neurological testing and brain scanning as these methods are expensive and machinery depended. There is no medication or cure is available to stop the progression and spreading of Parkinson's disease. Although, it is possible to stop or decrease the PD at earlier or infancy stages. But at later stages, when the disease has gone to be worst then an alternative solution is surgical treatment. However, for safety and cure the quality of patients brain cells, detection of Parkinsons disease at an earlier stage is necessary but clinical diagnosis are expensive, leads to inaccurate results and inadequate services to patients.

Over the period of time, a substantial number of studies based on handwriting have been presented and shown a product of perceptive, cognitive and fine motor skills that can also be employed as an effective tool for early diagnosis of PD [10-12]. Even though many clinical examinations as well as automatic diagnosis approaches PD have been proposed, it is still very important that we should exert more effort in automating its diagnosis efficiently. The paradigm-shifting results delivered by CNNs were in part accomplished with the help of extremely large training datasets, which is unrealistic Parkisons's identification due to availability of small data size, as a results, direct application of CNN could not be effective. In addition to the relatively small sample size, the other important limitation of most of the studies are focused on hand crafted features due to limitation of deep learning methods on small dataset, however, automatic extraction of features could helps to increase the identification performance [13]. Deep learning methods have demonstrated tremendous success in a variety of applications in various fields [14-19], however, it is data hungry approach and requires atleast 10 times the degree of freedom that can often preclude the use of CNNs for applications where dataset can be challenging. In order to address the problem of limited training data, transfer learning could be used to tune the already grained storing knowledge on similar problem. To overcome these challenges, in this paper, we plan to investigate the extendability of the trained CNN classifier on ImageNet and MNIST via transfer learning on target dataset named as PaHaW datset. The objective of this work is to perform an extensive experiment using Convolutional Neural Network (CNN) and the concept of transfer learning due to limited and finite samples of PaHaW Dataset collected from patients having PD and healthy persons, respectively. AlexNet is the relatively simple type of CNN architecture has got great interest and success in different pattern recognition and classification tasks.

In the literature, AlexNet has been proven as an excellent deep leaner for the various problem in different domains. Therefore, we explore the two common approaches of transfer leaning i.e., freeze and finetunining. In each approach, we conduct two studies. The first study uses pre-trained AlexNet on ImageNet dataset (natural images) and second study implement AlexNet form scratch on MNIST dataset (handwriting digits) as a source task for extraction of features for transferring to the target task PaaW datset. Each study has series of experiments for investigation of ways of features extraction like AlexNet-freeze and AlexNetfinetune due to limited samples of $\mathrm{PaHaW}$ dataset in the next sections.

The key contributions of this studies are :

- Development of early Parkinson's diagnosis using transfer learning and data augmentation techniques due to the limitation of handwritten data of Parkinson's patient.

- Find out the increase in the input raw samples using data-augmentation and other prepossessing techniques result in increasing the accuracy of deep CNN.

- Investigate that how the visual patterns of natural images (ImageNet dataset) and handwrittn digit images (MNIST dataset) can be benefitial in identification of PD.

- Explore that how features learned from one large dataset using freeze features and finetuning approaches of transfer learning can improve the recognition in other domain.

- This study analyze, evaluate and compare two different data sources for transfer learning for Parkinson's disease identification.

Rest of the paper is organized as follows. Section 2 demonstrates the concept of deep neural network and transfer learning in details. Section 3 presents the overview of related work regarding the deep and transferring based approaches for PD's identification. Section 4 describes the proposed methodology for Parkinson's diagnosis. Section 5 details the experimental results along with comparison with state-of-the- 
art work. Finally conclusion and future directions are discussed in Section 6.

\section{Related Work}

Ever since difficulties in handwriting were first reported by James Parkinson in patients with the shaking palsy, described as "the hand failing to answer with exactness to the dictates of the will". Earliest identification was based on paper based handwritten text of selected patient with significant observable micrographia [20]. Since then, Parkinson's disease identification an active area of research in the pattern recognition community for over four decades now. With the development of hand-held devices and digitizing tablet, the collection of handwriting samples using digitizing tablets is easy, non invasive and contains additional information (such as speed, pressure etc.) that paper based does not.

Until now, machine-learning based approaches are considered as supportive, and not substitutive of human in making the clinical decision. Unlike other machine learning applications, there are still some barriers to the complete translation in health industry. Recently, several attempts have been made to design decision support systems for differential diagnosis of PD in recent years. These includes speech assessment [21-27], gait monitoring [28-32] or tremor assessment $[33,34]$. There are several challenges involved in these methods i.e. speech assessment requires high quality noise free recording conditions, gait monitoring and tremor assessment requires specialized equipment such as accelerometers or gyroscopes. Whereas, handwriting based diagnosis of PD can be easily performed at clinic or even patient's home and does not require any special equipment for data acquisition. Previous studies have proven that there is significant difference between kinematics of PD patients and healthy controls. However extend to which any set of features could be useful in discriminating PD at early stage is still in progress.

Even though many automatic diagnosis approaches PD have been proposed, it is still very important that we should exert more effort in automating its diagnosis efficiently. In order to overcome limitation of conventional models the researchers moved towards deep learner in 2016. Pereira et al. [35] developed an handwriting dataset by capturing images during handwriting task and apply model on pen based features like pressure, tilt and acceleration. Convolutional Neural Network (CNN) was applied with image resolution for classification and achieved overall test set accuracy of $80.19 \%$ using ImageNet while considering spiral data. In an other work, Pereir et al. [36] applied CNN for identification of Parkinson's disease. Authors proposed meta-heuristic-based techniques that is Bat Algorithm (BA), Firey Algorithm (FA) and Particle
Swarm Optimization (PSO) in order to fine tune CNN hyper parameters.

Handwriting dataset has been used for detection of Parkinson's disease. CNN gave the effective results on BA approach with over all accuracy of $90.38 \%$ for spiral data. Pereira et al. map signals extracted from handwriting dynamics into images.These time series images passed to Convolutional neural network and achieved $93.50 \%$ accuracy using feature learned from CCN [37]. Zhang et al [38] applied stacked autoencoders and KNN classifier and employed the speech records and extract the time frequency features such as jitter, shimmer, voice pitch etc and got an accuracy $90.53 \%$. Grover et al. [39] applied Deep neural network using tensor flow library on voice dataset and yeilded $83.36 \%$ accuracy on Motor UPDRS Score and $94.42 \%$ accuracy on total UPDRS. Moetesum et al. [40] evaluated the visual attributes of handwriting for diagnosis of Parkinson's disease. Fusion techniques are applied in order to improve classification. For feature extraction applied the convolutional neural network and extracted features are then classify by SVM. Proposed method effectively diagnosed the Parkison's with 83\% accuracy. Caliskan et al. [27] proposed Deep neural network classifier for diagnosis of Parkinson's disease on two voice dataset i.e. Oxford Parkinson's Disease Detection (OPD) and Parkinson Speech Dataset with Multiple Types of Sound Recordings (PSD). Authors compared results of deep and conventional models. DNN has the ability to extract hidden features so as increase the classification performance. DNN classified the OPD and PSD dataset with effective accuracy of $93.79 \%$ and $68.05 \%$ whereas SVM , decision tree and naive Bayes has accuracy of 85.780, 84.371, 69.64\%.Eskofier et al. [32] performed the comparison of machine learning and deep learning techniques on IMU ( inertial measurement unit sensor) data for detection of Parkinson's disease. CNN effectively classified data with accuracy of $90.0 \%$ as compared to AdaBoost.M1, PART and kNN with accuracy of 86.3, 67.1 and $85.6 \%$ respectively. Choi et al. [41] developed an automatic deep learning-based FP CIT SPECT (I-fluoro propyl carbomethoxy iodophenylnortropane single-photon emission computed tomography) interpretation system for diagnosis of Parkinson's disease through images. They applied deep CCN for classification, proposed method have effectively diagnosed the Parkinson's with accuracy of 90.7\%. Afonso et al [42] used the different CNN architecture (Imagent,CIFAR10, LeNet) for classifying the recurrence plot images evaluated from signals of menders and spiral data. By using recursive approach, they have achieved the recognition rate above to $90 \%$. Gupta et al [43] proposed an optimized cuttlefish algorithm (OCFA )for feature selection evaluated it on Parkinson speech with multiple types of sound recordings and Parkinson Handwriting samples datasets. Decision tree and knearest neighbor classifier were used on OCFA se- 
lected feature that diagnose the Parkinsons disease with an accuracy of $94 \%$ approximately. Pereira et al. applied OSCA (optimal crow search algorithm) for feature selection from handwriting dataset and proposed Random Forest, Decision Tree, k-Nearest Neighbor classifier which gave the $100 \%$ prediction rate on OSCA selected features [44].

Not only handwritten text, Archimedean Spiral Drawing Test and shape modifications has also been used for Parkinson's diagnosis [28, 45-48]. Deviations from original sample (like loop tightness and width variability, drawing speed and acceleration, frequency and amplitude of oscillations and spiral pressure, etc.) are considered as symptomatic indicators of a disorder. Drotar et al. presented a template consisting of seven different handwriting tasks in addition to conventional spiral drawing task and suggested that the choice of template has significant impact on the performance of the proposed features [49].

TABLE 1: Summary of deep learning based methods for Parkinsons disease prediction

\begin{tabular}{|c|c|c|c|c|}
\hline Reference & Features & Method & $\begin{array}{l}\text { Data De- } \\
\text { scription }\end{array}$ & $\begin{array}{l}\text { Accuracy } \\
\%\end{array}$ \\
\hline $\begin{array}{l}\text { Pereira et } \\
\text { al (2016) }\end{array}$ & $\begin{array}{l}\text { pen base } \\
\text { features }\end{array}$ & CNN & \multicolumn{2}{|c|}{$\begin{array}{l}\text { Handwriting } 80.19 \\
\text { Data }\end{array}$} \\
\hline $\begin{array}{l}\text { Pereir et } \\
\text { al (2016) }\end{array}$ & $\begin{array}{l}\text { pen base } \\
\text { features }\end{array}$ & CNN & \multicolumn{2}{|c|}{$\begin{array}{l}\text { Handwriting } 90.38 \\
\text { Data }\end{array}$} \\
\hline $\begin{array}{l}\text { M Moete- } \\
\text { sum et al } \\
(2017)\end{array}$ & $\begin{array}{l}\text { automated } \\
\text { visual } \\
\text { features }\end{array}$ & CNN & \multicolumn{2}{|c|}{$\begin{array}{l}\text { Handwriting } 83 \\
\text { Data }\end{array}$} \\
\hline $\begin{array}{l}\text { Caliskan } \\
\text { (2017) }\end{array}$ & automated & DNN & Voice data & $\begin{array}{l}93.79 \\
\text { and } \\
68.05\end{array}$ \\
\hline $\begin{array}{l}\text { Eskofier } \\
(2016)\end{array}$ & automated & CNN & IMU Data & 90.9 \\
\hline Choi(2017) & automated & CNN & $\begin{array}{l}\text { SPECT } \\
\text { images }\end{array}$ & 90.7 \\
\hline $\begin{array}{l}\text { Pereira } \\
(2018)\end{array}$ & automated & CNN & $\begin{array}{l}\text { handwiting } \\
\text { data (time } \\
\text { series } \\
\text { image) }\end{array}$ & 93.50 \\
\hline $\begin{array}{l}\text { Zang } \\
(2013)\end{array}$ & $\begin{array}{l}\text { Time } \\
\text { frequency } \\
\text { features }\end{array}$ & $\begin{array}{l}\text { DNN } \\
\text { with } \\
\text { (KNN } \\
\text { ) }\end{array}$ & $\begin{array}{l}\text { Speech } \\
\text { dataset }\end{array}$ & 90.53 \\
\hline $\begin{array}{l}\text { Grover } \\
(2018)\end{array}$ & $\begin{array}{l}\text { Voice fea- } \\
\text { tures }\end{array}$ & DNN & $\begin{array}{l}\text { Voice Data } \\
\text { Set }\end{array}$ & $\begin{array}{l}94.42 \\
\text { and } \\
83.36\end{array}$ \\
\hline
\end{tabular}

The current systems are able to achieve about $90 \%$ accuracy. However, the unavailability of Parkisons's patient handwriting datasets and complexity of designing features are the major impediments preventing the research community from mastering this task. On the other-hand, recently deep learning approaches showed tremendous performance to deal such complex task by automatic learning of features as they learns features from raw data to a representation that best describe the data. Deep learning is good to learn visual features from any type of huge data without knowing which feature is important. Deep learning based techniques, however does not perform well on small datasets. It is greedy technique. ImageNet dataset is extensively used for the purpose of transfer learning for many image classification application due to its large size. However, Parkinson's disease dataset consists of handwritten samples, thus, MNIST could be the powerful source for transfer learning as compared to ImageNet. Therefore, In this paper, we are considering MNIST dataset as source dataset for features extraction and later compare with results achieved by networks using ImageNet dataet as a source.

\section{Deep Transfer Learning based Parkinson Identification System}

In this section, we demonstrate our proposed system using concepts of data augmentation, transfer learning and deep learning on handwriting sample images of Parkinson's patient. Even though, several automatic diagnosis methods for PD have been introduced, still it is very important to exert more effort in automating its diagnosis efficiently. The paradigmshifting results delivered by $\mathrm{CNNs}$ were in part accomplished with the help of extremely large training datasets, however, we do not have enough data of Parkinson's patient. Thus, direct application of CNN could not be effective on hundreds of images gathered by patients. To cope with this challenge, one way is the data augmentation. Number of CNN based networks are trained to investigate the potential of using the pre-trained CNN classifier on ImageNet and MNIST to learn features and then transfer the model on Parkinson's handwritten data. We have divided the proposed methodology into three steps i.e. Data preprocessing and data-augmentation, CNN based features extraction and identification.

Figure 1 shows the general overview of the proposed automated PD detection system using AlexNet with fine tune architecture and freeze architecture for transfer learning.

\subsection{Data Preprocessing and Data augmentation}

Pre-processing is a key step in pattern recognition and machine learning. Different techniques like binarization, transformation, image enhancement, sampling, normalization, data augmentation or noise removal etc. are the main techniques of preprocessing step that apply on raw data. As CNN directly works on raw data, thus, it needs to be refined before training to reduce the variations and noisy pattern. We conducted studies using PaHaw dataset having handwritten text written by PD's patients and healthy person. The original dataset has number of online attributes like $x, y$ coordinate, pen status (touching surface of paper 


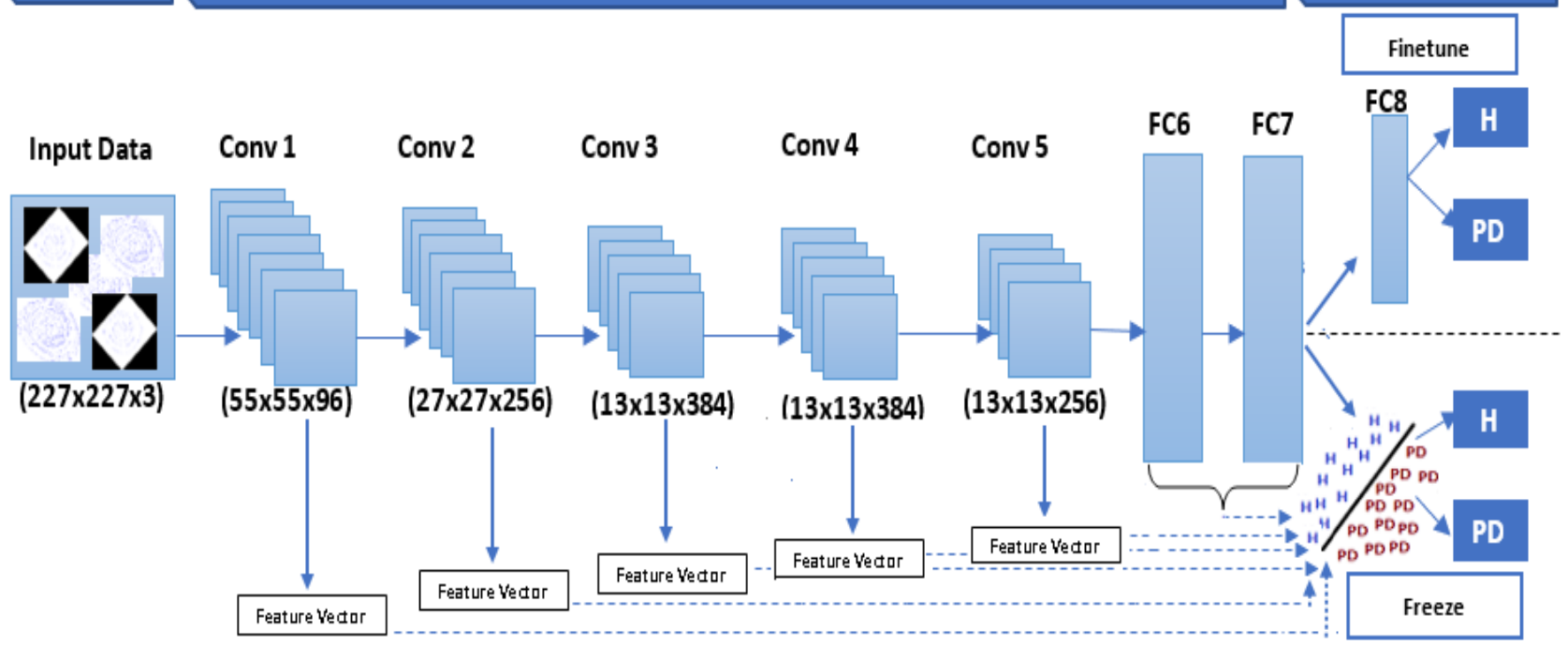

Figure 1: Overview of Proposed System for Parkinson's Disease Detection using AlexNet-Finetune and AlexNetFreeze

or not), pressure etc. We first plot the image using these information as shown in Figure.2.a. The handwritten text consist of several irrelevant information that could affect the accuracy of diagnosis i.e., $\mathrm{PaHaW}$ data contain values of in-air as well as on surface movement. We have removed the in-air movement values from images. Figure.2.b depicts the removal of in-air movement. We can observe from the data that the handwritten text consist of extensive amount of noise, thus, we have removed the noise from data first by applying filters such as median filter followed by transferring it into gray scale.

Deep neural networks need large number of instances of images for training the networks, however, in our case, we do not have enough data of Parkinson's patient. Thus, direct application of CNN could not be effective on hundreds of images collect of Parkinson's patients. To cope with this challenge, one way is the data augmentation that can significantly improved the performance of Deep Neural networks. Therefore, we have applied several data-augmentation techniques (rotations, flipping and contours) to increase the the $\mathrm{PaHaW}$ training dataset for providing large input space to CNN. In order to reduce over-fitting and increase generalization of network, we deployed data augmentation to collect variety of visual patterns of input raw images as in our case we have small number of handwritten samples from Parkinson's patients. The basic Data augmentation techniques applied in this study like rotation and flipping. The input images are rotated on various angle like angle of 45, 90, 135, 180, 225, 270, 315 and 360. Likewise, input images are

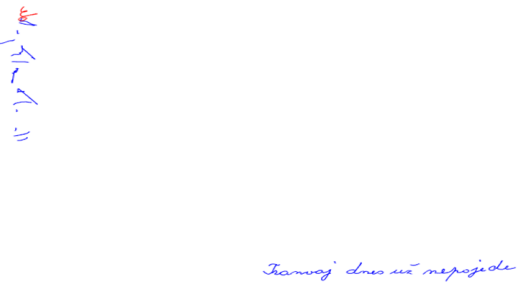

(a) Sample of original image

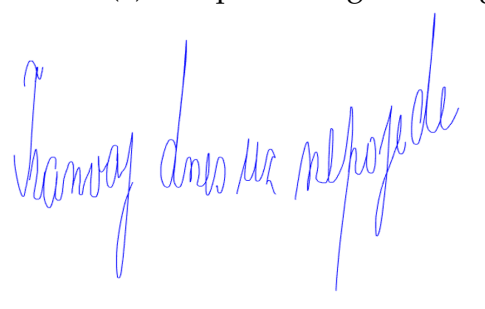

(b) Pre-processed image

Figure 2: Removal of irrelevant information and white region

horizontally and vertically flipped. As a result, we got 10 variations of one original image using basic techniques of data augmentation. For useful and effective extraction of visual patterns, other techniques are also used for increasing the size. Besides basic data augmentation techniques, we also created contours, high frequency, sharpness of image for expanding the size of dataset. Contours are a single unit thickness of a pattern which help in good features extraction. Con- 
tours are computed in the handwritten domains for the effective results. Thus, we computed the contour using $C(x, y)=255-\operatorname{Image}(x, y)$.

High frequency is computed with the aid of discrete Fourier transform, or DFT. The foundation of the product is the fast Fourier transform (FFT), a method for computing the DFT with reduced execution time. For the input sequence ' $\mathrm{x}$ ' and its transformed version ' $X$ ' (the discrete-time Fourier transform at equally spaced frequencies around the unit circle), the two functions implement the relationships:

$$
X(k+1)=\sum_{n=0}^{N-1} x(n+1) W_{N}^{k n}
$$

and

$$
x(n+1)=\frac{1}{N} \sum_{k=0}^{N-1} X(k+1) W_{N}^{-k n}
$$

At final step, we use unsharp masking in order to sharp the original image. The sharp image is generated by simply subtracting the image form blurred version of itself as $S_{h p}(x, y)=\operatorname{Image}(x, y)-\operatorname{Image}_{l p}(x, y)$; where $h p$ is the high pass filter and $l p$ is the low pass filter. The size " $N$ " of $\mathrm{PaHaW}$ dataset has increased to "13 $x N^{\prime \prime}$ augmented dataset.

In order to train AlexNet on MNSIT (base task) from scratch, we have performed other preprocessing approaches like resizing and channelization of digit images. As an image of MNIST dataset has 28X28 dimension so we have to re-size it on 224X224 dimension according to AlexNet architecture and have to convert from $2 \mathrm{D}$ into $3 \mathrm{D}$. It means that we converted gray scale (1 channel) images to RGB (3 channel image) for scratch training of AlexNet architecture then we can use the visual patterns extracted from digits automatically by CNN and transfer to our augmented target task (PaHaW dataset) in classification and identification step.

In next section, the approaches of transfer learning like AlexNet-freeze and AlexNet-finetune are deploying for features extraction form source datasets i.e., ImageNet and MNIST datasets.

\subsection{CNN based features extraction}

The Convolutional Neural Networks (CNN) are biologically-inspired variants of Multilayer Perceptrons (MLPs) that perform machine learning tasks without requiring any handcrafted feature to be engineered and supplied by the user. Recently, have gained considerable commercial interest due to the development of new variants of CNNs and showed promising performance by advocating the superiority over traditional machine learning algorithms. The main power of a CNN lies in its deep architecture, which allows for extracting a set of discriminating visual features at multiple levels of abstraction. They have different kinds of layers and each layer works different than other for extraction of visual features. However, one of the biggest limitation is the unavailability of larger and labeled good quality data. The availability of large datasets is rare in the field of healthcare, as data in health industry is very sensitive, expensive confidential and very hard to collect.

Due to insufficient amount of training data, we have implemented pre-trained AlexNet architecture of CNN [50] for extraction of features. In the literature, this technique is known as transfer learning. The visual features are extracted in two ways i.e., a pretrained AlexNet with freeze or fixed approach and pre-trained AlexNet with fine-tune approach. Previous studies have clearly demonstrated that the selection of the source task has an great impact on the performance of $\mathrm{CNN}$ on the target task. Recently, ImageNet has been adopted as source for Parkinson's diagnosis [10]. However, MNIST dataset has not explored for features extractions in transfer learning domain for PD identification in the literature. It can provide better performance due to its relevance to the data of PD dataset as both datasets have handwritten text samples.

We deployed the 25 layered Deep Convolutional Neural Network for features extraction and then transfer these features to learn visual patterns from handwriting samples of PaHaW dataset for identification of PD using SVM classifier. AlexNet architecture consists of 5 convolution layers, max-pooling layers, dropout layers, and three fully connected layers.The output of fully connected layer is pass to 1000 -way which yields a distribution across 1000 class labels. First convolutional layer filters the $227 \times 227 \times 3$ input images with 96 kernels that have size $11 \times 11 \times 3$ with 4 pixels stride. Second convolutional layer takes the output of first layer (response-normalized and pooled) as an input and filters it with 256 kernels of $5 \times 5 \times 48$ size. Others convolutional layers are connected to each others without any intermediate pooling or normalization layers. Third layer has 384 kernels having $3 \times 3 \times 256$ connected to the normalized and pooled output of second layer whereas fourth layer contains 384 kernels of size $3 \times 3 \times 192$ and fifth layer has 256 kernels of size $3 \times 3 \times 192$. Fully connected layers contains 4096 neurons each. The convolutional layers have more general weights and features which become more specific on fully connected layers. The detailed of AlexNet architecture deployed in our experiments are depicted in Table 3.

The network constructs a hierarchical representation of input images. Deeper layers includes higher-level features, constructed using the lower-level features of earlier layers. Together, the convolutional and down sampling layers serve as feature extractors while the fully connected layers represent a trainable classier similar to a standard multi-layer neural network. In this paper, we conducted two studies 


\section{TABLE 2: AUGMENTATIONNNNn}

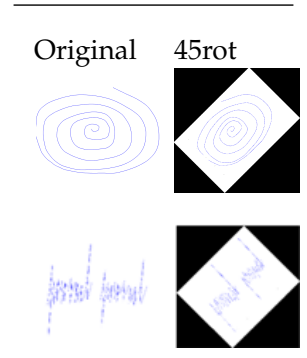

TABLE 3: The detailed AlexNet Architecture used in our Experiments.

\begin{tabular}{cccccc}
\hline Layer & Type & Input & Filter and Size & Stride & Output \\
\hline Data & Input data & $227 \times 227 \times 3$ & - & - & $3 \times 227 \times 227$ \\
Conv1 & convolution & $227 \times 227 \times 3$ & $11 \times 11$ and 96 & 4 & $55 \times 55 \times 96$ \\
pool1 & max pooling & $55 \times 55 \times 96$ & $3 \times 3$ & 2 & $96 \times 27 \times 27$ \\
Conv2 & convolution & $27 \times 27 \times 96$ & $5 \times 5$ and 256 & 2 & $27 \times 27 \times 256$ \\
pool2 & max pooling & $27 \times 27 \times 256$ & $3 \times 3$ & 2 & $256 \times 13 \times 13$ \\
Conv3 & convolution & $13 \times 13 \times 256$ & $3 \times 3$ and 384 & 1 & $13 \times 13 \times 384$ \\
Conv4 & convolution & $13 \times 13 \times 384$ & $3 \times 3$ and 384 & 1 & $13 \times 13 \times 384$ \\
Conv5 & convolution & $13 \times 13 \times 384$ & $3 \times 3$ and 256 & 1 & $13 \times 13 \times 256$ \\
poo15 & max pooling & $13 \times 13 \times 256$ & $3 \times 3$ & 2 & $256 \times 6 \times 6$ \\
Fc6 & fully connected & $6 \times 6 \times 256$ & $6 \times 6$ and & - & $4096 \times 1$ \\
Fc7 & fully connected & $4096 \times 1$ & $1 \times 1$ and & - & $4096 \times 1$ \\
Fc8 & fully connected & $4096 \times 1$ & $1 \times 1$ and & - & $2 \times 1$ \\
Output & fully connected & $2 \times 1$ & - & - & $2 \times 1$ \\
\hline
\end{tabular}

having two different source dataset. One study explored the effect of visual patterns from ImageNet and another study carried out to investigate the impact of visual patterns extracted from MNIST dataset as the source tasks. The source tasks that the ImageNet dataset consists of 1.2 million images (1000 different classes) in one study and MNIST consists of 0.6 million images (with 10 different classes) in another study for extraction of visual features. The target task is PaHaW dataset having 598 images (75 subject and 2 target classes) for identification of PD. These studies for AlexNet based feature learning, i.e, reusing of freeze or fixed features based approach and fine-tuning the features based approach are demonstrating in the following subsections.

3.2.1. Reusing of Fixed or Freeze Features. We use the weights derived from training the network on the source task (ImageNet and MNIST datasets) and using the outputs from the intermediate hidden layer (like edges and blobs) as features for training a linear classifier on the data of target task (PaHaW dataset). This is called reusing freezing or fixed approach of transfer learning. We have convolutional (conv) and fully connected $(\mathrm{fc})$ layers from which the features can be derived. The layers named as conv1, conv2, conv3, conv4, conv5, fc6 and fc7 in AlexNet's architecture, respectively.

In this study, we carried out series of experiments that which layer extract best features for our target dataset using tow different source datasets. The different layers of AlexNet are treated as fixed feature vectors extracted using ImageNet or MNIST database independently. Then fed to another linear classifier for classification and identification PD using PaHaW dataset. We conducted number of experiments to explore that which layer outperforms in extraction of features as compare to other. We employed transfer learning by extracting features from different layers like conv1-conv5, fc6, fc7 and fusion of $\mathrm{fc} 6+\mathrm{fc} 7$ of the architecture of AlexNet then fed these features to a dedicated linear classifier i.e. SVM for classification as depicts in Figure 1. The deeper we go into the model, the weights represent more minute and smaller details of an image. Thus, conv1-conv5 show higher level and generic representation of source dataset's images (like edge, blob etc.) while outputs of fc7 represent a more detailed and specific features of the images of source dataset.

\section{TABLE 4: The Dimensions of Features Vectors}

\begin{tabular}{llllllll}
\hline Conv1 & Conv2 & Conv3 & Conv4 Conv5 fc6 & fc7 & fc6+fc7 \\
\hline 290400 & 186624 & 64896 & 64896 & 43264 & 4096 & 4096 & 8,192 \\
\hline
\end{tabular}

3.2.2. Fine-Tuned Features. This technique works by transfering the weights of the pre-trained model using two source dataset (ImageNet or MNIST) to a network 
using target dataset (PaHaW). The only exception is the replacement of last fully connected layer of the pretrained network with new network's fully connected layer. The new last layer has the same number neuron as of the target classes of the target dataset or task. This is called fine tuning approach of transfer learning.

By implementing these technique, we conducted two studies. First, we have employed pre-trained AlexNet for feature extraction using ImageNet dataset (source task), then replaced the last fully connected layer having 1000 neurons from ImageNet with fully connected layer consisting of 2 numbers of neurons from $\mathrm{PaHaW}$ dataset (target task).

In second study, experiment carried out using MNIST dataset as a source task and PaHaW dataset as target task. As the last layers of pre-trained AlexNet are configured for 1000 classes of ImageNet datset so we have to replace and fine tune the last fully connected layer of AlexNet architecture to our new problem. We have set the fully connected layer to two classes in our new dataset i.e. Parkinson's Diseases $(\mathrm{PD})$ or healthy $(\mathrm{H})$ for classification and identification purpose as shown in figure 1.

\subsection{Classification and Identification}

Once the AlexNet trained for Parkinson Disease (PD) learning and classification, then unseen PD and Healthy $(\mathrm{H})$ image(s) in the test set are fed to trained network for identification. The maximum number of epochs are 30. The training network takes 148 iterations per one epoch and total iterations for whole training are 4440 . The values of momentum, L2 regularization, initial learn rate and batch size are 0.9, $0.0005,0.0001$ and 7. Total elapsed time is taken upto 10 minutes and 34 second for training a network on fine-tuned based features using ImagNet dataset for PD identification from $\mathrm{PaHaW}$ dataset.

The highest recognition rate $(98.28 \%)$ achieved on spiral pattern using AlexNet-Finetune-ImageNet approach of transfer learning as depicted in Figure. 3. In the subsequent section, a detailed experimental analysis for parameters, patterns and comparative analysis are given with the existing PD identification system in the literature.

\section{Experimental Design, Analysis of Results and Discussion}

This section aims at presenting the experimental designs and results analysis concerning the CNNbased approach for Parkinsons Disease identification. we evaluate the different parameters for getting the best values of parameters for our experiment, evaluate the CNN networks on different patterns and different approaches (Freeze and Finetune) of transfer learning using ImageNet and MNISt datasets and compare our approach with other state of the art methods $[35,36,40]$.

\subsection{Study Subjects}

We used PaHaW (Parkinson's disease handwriting) database to evaluate our proposed studies. It includes the handwriting samples of 37 Parkinsons patients and 38 healthy subjects, that collected by Droter [49] using digitized tablets. The mean UPDRS-Part V score for PD patients is $2.27 \pm 0.84$. Each participants asked to perform total eight handwriting tasks but some participants did not completed their task according to given sample, hence, we have excluded their samples. After exclusion, we have 576 samples from 72 (36 $\mathrm{PD}$ and 36 control) subjects. The $\mathrm{PaHaW}$ is collecting using digitizing tablet, thus it consists of various online attributes such as the $(x, y)$ coordinates of the pen trajectory as well as the pen status (whether touching the writing surface or in air) rather than images. We have generated images of the drawing by plotting the normalized $(\mathrm{x}, \mathrm{y})$ coordinates corresponding to all positions where the pen is touching the writing surface.

The idea of capturing more information from different samples produced by the same subject was the prime incentive for using $\mathrm{PaHaW}$ i.e. dataset consist of different task (tas1-task8) perfromed by same subject. First task contain spiral drawings [51] [52] because it is continuous handwriting sample and it is most suitable for evaluation of movement disorder. Another tasks includes the repeated cursive letter " 1 " and simple words and a complete sentence. An example of samples of dataset are shown in Figure 4. Description and statistics of data set as illustrated in table 5. The $\mathrm{PaHaW}$ dataset is distributed in training and testing set for performance of our proposed methodology.

\section{TABLE 5: The PaHaW dataset description}

\begin{tabular}{|l|l|l|l|l|}
\hline Task & Samples & PD & Healthy & Instances \\
\hline 1 & ArchimedeanSpiral & 36 & 36 & 72 \\
\hline 2 & Letter 'l' $^{\prime}$ & 37 & 38 & 75 \\
\hline 3 & Bigram 'le $^{\prime}$ & 37 & 38 & 75 \\
\hline 4 & Word 'les' $^{\prime}$ & 37 & 38 & 75 \\
\hline 5 & Word 'lektorka' $^{\prime}$ & 37 & 38 & 75 \\
\hline 6 & Word 'porovnat' $^{\prime}$ & 37 & 38 & 75 \\
\hline 7 & Word 'nepopadnout' & 37 & 38 & 75 \\
\hline 8 & Sentence & 37 & 38 & 75 \\
\hline \hline
\end{tabular}

\subsection{Experimental results}

Alexnet is trained by varying the options of training parameters to get highest accuracy by training number of networks for our dataset using single Graphics Processor Unit (GPU). We used the implementations provided by the well-known Caffe library7, which is developed under a General-Purpose 


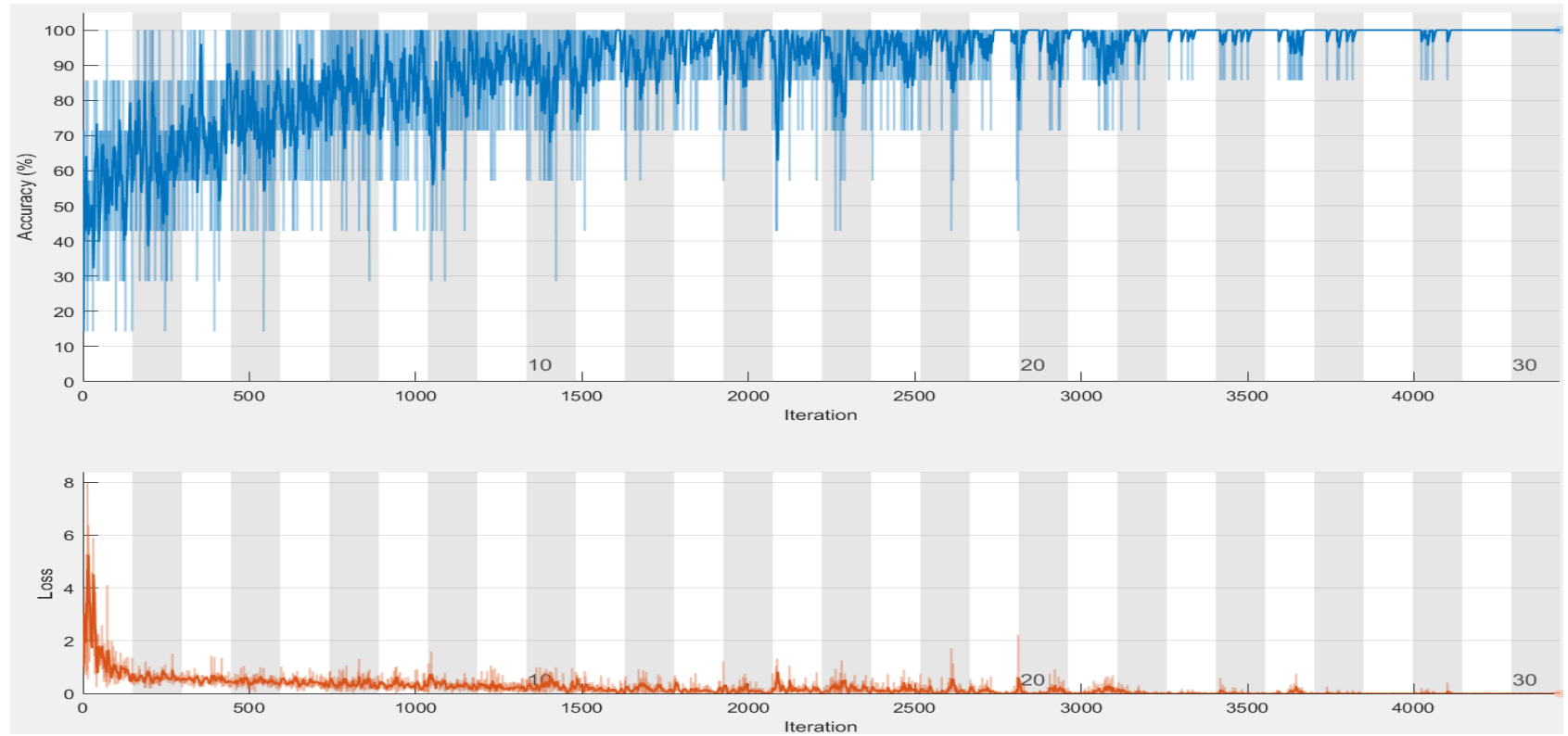

Figure 3: The training progress and error rate of the proposed system for Parkinson's disease detection using AlexNet-Finetune-ImageNet approach

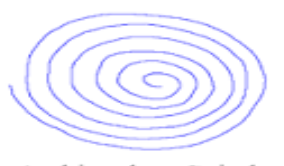

Archimedean Spiral

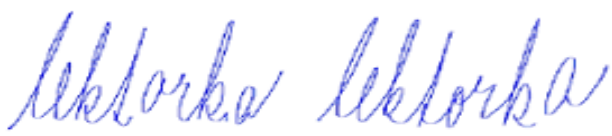

Word 'lektorka'

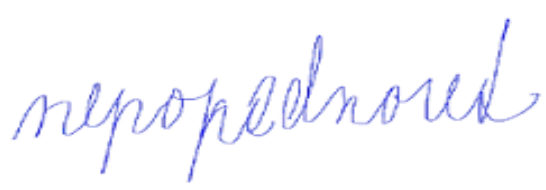

Word 'nepopadnout'

Letter ' 1 '

(1)

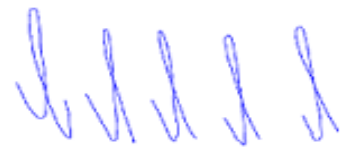

Figure 4: An example of
sits using Single

GPU having multiple processor of $2.80 \mathrm{GHz}$ and 2.81 GHz. By default parameters are 0.9 momentum, 128 batch size, 0.0005 L2 regularization, 30 epochs, positive scalar for initial learning rate, 10 learn rate drop period and 0.1 learn rate drop factor. We have conducted number of experiments for evaluation of proposed CNN based system's performance. We kept all default parameters and evaluate the performance of proposed fine-tuned features based system by changing the values of the momentum, Initial Learn Rate, L2 regularization and batch size. The best network achieved
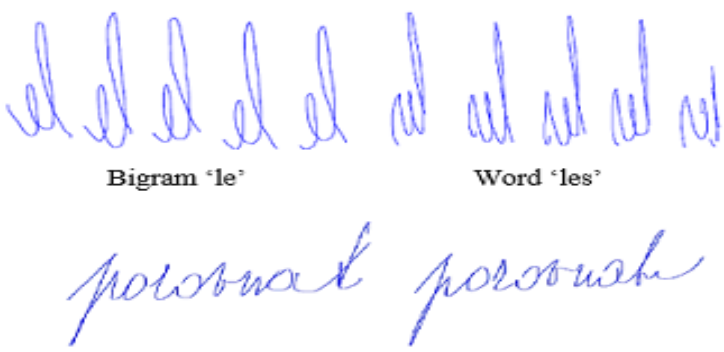

Word 'porovnat'

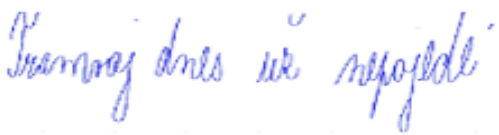

Sentence

samples of $\mathrm{PaHaW}$ dataset

using 0.9 momentum, $0.0005 \mathrm{~L} 2$ regularization, 0.0001 initial rate and 7 batch size and got best results upto $98.28 \%$. The best parameter values in our study are shown in Table. 6.

As discussed in earlier section, in this paper, two transfer learning techniques (Freeze and Fine-tuned) are carried out using augmented $\mathrm{PaHaW}$ dataset [35] consisting of eight handwritten patterns collected by Parkinson patients and healthy subjects. We have divided the dataset into training set that consist of $90 \%$ of samples and testing dataset that consist of $10 \%$ samples. 
TABLE 6: Parameters values for fine-tune training the network by using features extracted by CNN

\begin{tabular}{|c|c|c|c|c|c|c|c|c|c|c|c|c|c|c|}
\hline Parameters & \multicolumn{14}{|c|}{ Values } \\
\hline Momentum & 0.9 & 0.7 & 0.7 & 0.7 & 0.7 & 0.8 & 0.9 & 0.9 & 0.9 & 0.9 & 0.9 & 0.9 & 0.9 & 0.9 \\
\hline L2 Regularization & 0.0005 & 0.00005 & 0.0005 & 0.0005 & 0.00005 & 0.005 & 0.0005 & 0.0005 & 0.0005 & 0.0005 & 0.0005 & 0.0005 & 0.0005 & 0.0005 \\
\hline Initial Learn Rate & 0.001 & 0.001 & 0.0001 & 0.0001 & 0.0001 & 0.1 & 0.0001 & 0.0001 & 0.0001 & 0.0001 & 0.0001 & 0.0001 & 0.0001 & 0.0001 \\
\hline Batch Size & 128 & 128 & 128 & 7 & 128 & 100 & 255 & 200 & 128 & 100 & 64 & 32 & 7 & 1 \\
\hline Time (min: & $7: 23$ & $7: 41$ & $7: 35$ & $11: 38$ & $7: 39$ & $7: 31$ & $7: 53$ & $7: 25$ & $7: 48$ & $7: 31$ & $7: 39$ & $7: 43$ & $10: 54$ & 32.10 \\
\hline Accuracy (\%) & 51.72 & 65.52 & 73.28 & 79.31 & 53.45 & 46 & 92.24 & 92.24 & 95.60 & 95.69 & 93.10 & 93.10 & 98.28 & 50 \\
\hline
\end{tabular}

To explore the performance of Freez based transfer learning, first we explore the performance of AlexNet on different patterns and combined data of $\mathrm{PaHaW}$ dataset. Series of eighteen experiments were conducted by fixing conv5 layer using AlexNet-freezeImageNet features and AlexNet-freeze-MNIST features, respectively. In the first nine experiments, we investigated and evaluated each and every pattern of PaHaW dataset independently using Alexnet-freeze by fixing the conv5 layer on ImageNet dataset and then other nine series of experiments performed on AlexNet-freeze (conv5) using MNIST dataset. In Table 7, performance of each pattern of $\mathrm{PaHaW}$ dataset using fixed conv5 is further demonstrated and compared with both features. Results show that, using AlexNet-freeze-ImageNet, the pattern of spiral drawing (pattern-1) has the highest recognition rate as compare to other patterns. Next higher identification rate is achieved on two short word (pattern-6) are $89.29 \%$ and then $79.49 \%$ on two letter word (pattern-3) . Similarly, the results are $76.72 \%, 72.32 \%, 73.21 \%$ and $78.99 \%$ for patterns-2, patterns- 5 , patterns- 4 and pattern-7 respectively. The proposed pre-trained AlexNet-freezeImagenNet network gave $77.48 \%$ recognition rate on full sentence (pattern 8), whereas it provided $84.19 \%$ identification rate on combined patterns. The evaluation of each pattern using AlexNet-freeze-MNIST features is demonstrated in Table 7. Result shows that pattern of spiral drawing (pattern-1) provided the highest recognition rate of $94.97 \%$ as compare to other patterns and the next higher identification rate achieved on sentence (pattern-8) are $86.49 \%$ and $86.32 \%$ on two letter word (pattern-3) . Similarly, $86.21 \%, 77.68 \%, 81.25 \%$ and $83.19 \%$ for patterns2,patterns-4,patterns-5 and pattern-7 respectively. The proposed pre-trained AlexNet-freeze-MNIST network provided $68.75 \%$ recognition rate on two short word (pattern 6) and overall classification $77.28 \%$ on all patterns.

In the second technique (Fine-tuned) of transfer learning, we have also trained eighteen networks using AlexNet-finetune features for different patterns of $\mathrm{PaHaW}$ dataset. We conducted two studies to explore either which pattern or combined data show the best performance based on AlexNet-finetune features from sources i.e., ImageNet, MNIST datasets or independently (in last two columns of Table 7). The networks showed promising accuracy for spiral drawing of pattern-1 using fine-tuned features from ImageNet $(98.28 \%)$ and MNIST $(80,17 \%)$ as compare to other patterns. The performance of networks are not satisfactory on combined data of all patterns as $84.10 \%$ achieved by network trained AlexNet-finetune-ImageNet features and $77.28 \%$ accuracy got by network trained on MNIST dataset using fine-tuning approach .

We can conclude from the above discussion that accuracy differ for different pattern using AlexNetfreeze-ImageNet, AlexNet-freeze-MNIST, AlexNetfinetune-ImageNet and AlexNet-finetune-MNIST features. Notice that, all features provided promising result on spiral pattern as compare to others. Spiral images are informative in case of Parkinson's disease detection so we conducted further experiments on spiral pattern. Table 8 depicts the average of ten runs for Parkinson's disease identification rate for the series of experiments having fixed features of con1-conv5, fc6, fc7, fusion of fc6 and fc7 and fine-tuned features using ImageNet dataset as source task and spiral pattern of $\mathrm{PaHaW}$ dataset as target task. $93.10 \%$, $93.97 \%, 94.83 \%, 96.69 \%$ and $98.28 \%$ accuracy achieved using conv5, fc6, fc7, fc6 $+\mathrm{fc}_{\mathrm{c}} 7$ and fine-tuned based features using ImageNet and $\mathrm{PaHaW}$ dataset (spiral), respectively. The Table 9 also shows the precision, sensitivity and specificity as the evaluation matrices for these experiments.

In case of reusing of fixed features case, it is concluded from series of experiments that highest accuracy $96.69 \%$ is achieved on learned features from fusion of fc6 and fc7 using ImageNet dataset. The results are less in other experiments having fixed features of fc7, fc6 and con1-conv5. as shown in table 8 and table 9. Similarly, AlexNet-freeze based features achieved $94.97 \%, 91.38 \%, 90.52 \%$ and $92.24 \%$ accuracy on MNIST using conv5, fc6 ,fc7 and fc6+fc7, respectively as shown in table 8 and table 10 . The highest results up to $94.97 \%$ achieved using AlexNet-freezMNIST at conv5 layer. While, Alexnet-finetuned based learning achieved the accuracy of $80.17 \%$ accuracy on MNIST dataset.

Fine-tune approach shows interesting results for both ImageNet and PaHaW datasets. AlexNetfinetune-ImageNet features yield outstanding result $98.28 \%$ due to large size of ImageNet dataset and 1000 classes. We assumed that MNIST dataset will perform well as compare to ImageNet dataset but AlexNetfinetune-MNIST network showed unsatisfactory per- 
TABLE 7: Results on each pattern using AlexNet-freeze-ImageNet features, AlexNet-freez-MNIST, AlexNetfinetune-ImageNet and AlexNet-finetune-MNIST on augmented PaHaW dataset

\begin{tabular}{|l|l|l|l|l|l|}
\hline Pattern & Samples & $\begin{array}{l}\text { ImageNet conv5 fixed Fea- } \\
\text { tures based Accuracy (\%) }\end{array}$ & $\begin{array}{l}\text { MNIST conv5 fixed Fea- } \\
\text { tures based Accuracy (\%) }\end{array}$ & $\begin{array}{l}\text { Finetuned-ImageNet } \\
\text { Accuracy (\%) }\end{array}$ & $\begin{array}{l}\text { Finetuned-MNIST } \\
\text { Accuracy (\%) }\end{array}$ \\
\hline pattern-1 & spiral & $\mathbf{9 3 . 1 0}$ & $\mathbf{9 4 . 9 7}$ & $\mathbf{9 8 . 2 8}$ \\
\hline pattern-2 & Letter & 76.72 & 86.21 & 91.38 \\
\hline pattern-3 & twoletterword & 79.49 & 86.32 & 96.58 \\
\hline pattern-4 & threeletterword & 73.21 & 77.68 & 70.17 \\
\hline pattern-5 & shortword & 72.32 & 81.25 & 71.79 \\
\hline pattern-6 & twoshortword & 89.29 & 68.75 & 63.93 \\
\hline pattern-7 & longwords & 78.99 & 83.19 & 75.00 \\
\hline pattern-8 & sentence & 77.48 & 86.49 & 53.19 \\
\hline all patterns & all samples & 87.05 & 74.53 & 62.50 \\
\hline
\end{tabular}

TABLE 8: Parkinson's disease identification (ImageNet-PaHaW and MNIST-PaHaW)) using different architecture of AlexNet: Reusing of Freeze layers and Fine-tune approaches of transfer learning on Pattern-1

\begin{tabular}{|l|l|l|}
\hline & \multicolumn{2}{|l|}{ Accuracy \% } \\
\hline Experiment & ImageNet & MNIST \\
\hline conv1 & 49.14 & 50.00 \\
conv2 & 45.69 & 74.14 \\
conv3 & 54.31 & 93.62 \\
conv4 & 62.07 & 92,76 \\
conv5 & 93.10 & $\mathbf{9 4 . 9 7}$ \\
fc6 & 93.97 & 91.38 \\
fc7 & 94.83 & 90.52 \\
fc6+fc7 & $\mathbf{9 6 . 6 9}$ & 92.24 \\
Fine Tuning & $\mathbf{9 8 . 2 8}$ & 80.17 \\
\hline \hline
\end{tabular}

formance and achieved $80.12 \%$ due to small size of data and 10 classes digits. Results showed that fine tuning of the ConvNet is applicable in our case due to the over-fitting concerns on MNIST daatset. From the results, we notice that in case of small and different dataset like $\mathrm{PaHaW}$, it is the best choice to train a linear classifier on extracted freeze features from source datset.

TABLE 9: Performance evaluation of Parkinson's disease identification using different fixed layers in Freeze approach and Fine tuning approach of Transfer learning using ImageNet and $\mathrm{PaHaW}$ datasets

\begin{tabular}{l|lll}
\hline \hline Experiment & Precision & Sensitivity & Specificity \\
\hline \hline conv5 & 91.23 & 82.30 & 91.83 \\
fc6 & 94.55 & 89.66 & 94.83 \\
fc7 & 95.93 & 90.31 & 95.10 \\
fc6+fc7 & 97.21 & 91.55 & 98.16 \\
Fine tuning & 85.98 & 67.57 & 76.37 \\
\hline \hline
\end{tabular}

\subsection{Validation of Dataset}

Cross-validation is a statistical method that is applied for the performance evaluation of predictive model on an unknown dataset. We conducted the kfold cross validation on single task of dataset i.e. spiral task only. The reason behind $\mathrm{k}$-fold cross validation is
TABLE 10: Performance evaluation of Parkinson's disease identification using different fixed layers in Freeze approach and Fine tuning approach of Transfer learning using MNIST and PaHaW datasets

\begin{tabular}{l|lll}
\hline \hline Experiment & Precision & Sensitivity & Specificity \\
\hline \hline conv5 & 94.74 & 93.10 & 94.83 \\
fc6 & 93.55 & 88.66 & 93.83 \\
fc7 & 92.73 & 87.93 & 93.10 \\
fc6+fc7 & 88.89 & 96.55 & 87.93 \\
Fine tuning & 61.62 & 54.91 & 61.98 \\
\hline \hline
\end{tabular}

that, it guarantees that each sample eventually become the part of training as well as testing sets.The spiral sample contains 936 images. First, we divide the data into 4 folds. We have conducted the experiment in such a way that 234 images is reserved for testing and remaining 702 images in 3 folders are used for training purpose. The learning model is then trained on 3 subsets (training set) and then model is tested on the remaining subsets (test or validation set).

The overall split of the dataset for 4-fold cross validation and the results obtained by 4 -fold cross validation are shown in Figure. 5. The average identification rate of 4-fold cross validation using fine-tuned features of ImageNet is computed as $98.28 \% \pm 0.38$.

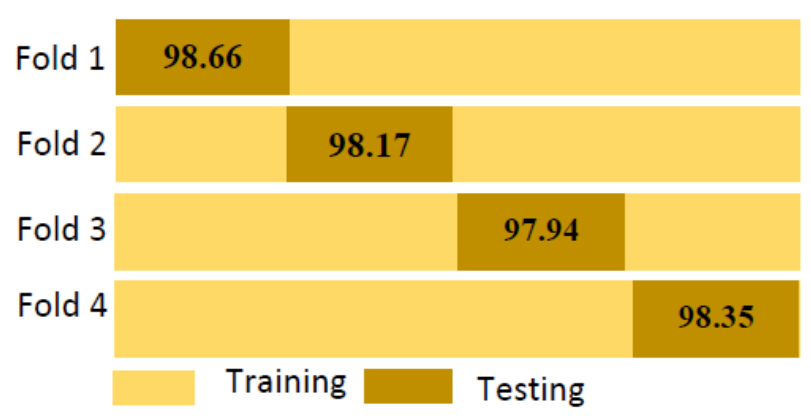

Figure 5: 4-fold cross validation 


\subsection{Evaluation Matrix}

In order to evaluate the performance of proposed Parkinson's disease classification system, we have used sensitivity, specificity and precision as evaluation measures and performed 4-fold evaluation. Each terminology is expressed by True Positives (tp), False Positives (fp), True Negatives (tn) and False Negatives (fn) rates.

- Accuracy determines the overall ability of the system to correctly classify data (PD and healthy subjects)

$$
\text { Accuracy }=\frac{t p+f n}{t p+t n+f p+f n}
$$

- Precision is the true positive relevant measure and is calculated by

$$
\text { Precision }=\frac{t p}{t p+f p}
$$

- Sensitivity determine the ability of system to correctly classify the PD subjects and is defined as the proportion of True Positives in the diseased cases and calculated by

$$
\text { Sensitivity }=\frac{t p}{t p+f n}
$$

- Specificity determines the ability of the system to correctly classify the healthy subjects and is claculated by

$$
\text { Specificity }=\frac{t n}{t n+f p}
$$

Tables 9 and 10 depict the precision, sensitivity and specificity of various layers of Alexnet-freez-Imagenet and Alexnet-freez-MNIST. These tables aslo precision, sensitivity and specificity of the proposed systems using fine-tune approach of transfer learning using ImageNet and MNIST.

\subsection{Discussion}

In this section, we analyzed and compare the performance of proposed system with existing state of the art Parkinson's classification systems on PaHaW as target dataset using ImageNet and MNIST as source dataset. Table 11 describes the comparison of different Parkinson's disease classification.

A meaningful comparison of our system is possible with works of Pereira et al. [35, 36] and Moetesum et al [10]. Pereira et al. [35] applied CNN on pen based features by using three different techniques. We compare our system with his ImageNet technique outperform their work by achieving $98.19 \%$ in comparison to $80.19 \%$ using ImageNet data on time series data. In another work, they have applied meta-heuristic-based techniques that is Bat Algorithm (BA), Firey Algorithm
(FA) and Particle Swarm Optimization (PSO) in order to fine tune CNN hyper parameters [36]. Handwriting dataset has been used for detection of Parkinson's disease and provided effective results on BA approach with over all accuracy of $90.38 \%$ for spiral data. In 2018, Pereira et al. [37] combined six representations ( spirals, drawing of circles on the page and in air, meanders, left-wrist and right-wrist movements) and extracted features by deploying $\mathrm{CNN}$ using pen based features (time series) from online images and reported about $93.50 \%$ accuracy. In both studies, time series data was used. In comparison to Pereira et al. [35-37], our system showed consider gain in performance i.e. $80.19 \%$ [35], $90.38 \%$ [36], and 93.50 [37] to $98.28 \%$.

Moetesum applied pre-trained AlexNet-freezeImagNet for feature extraction using three representations of offline images in input space and then apply SVM for classification using visual attributes yielded $83 \%$ [10]. However, in this work, we have applied the same model but further explore the performance of $\mathrm{CNN}$ model named as AlexNet architecture for different patterns of $\mathrm{PaHaW}$ dataset and investigated thoroughly the three common approaches i.e., scratch, freeze or fixed features and fine-tune features. Results of [10] showed that basic approach showed poor performance. To improve the classification performance, we have used two different source datasets (ImageNet and MNIST) and their impact on PD identification. To increase the size of training dataset, we have used different techniques of data augmentation (rotation, fliping, contour etc) with raw images. In reusing of freeze features of transfer learning, we thoroughly explored features at each layer and fusion of fc6 and fc7. The conv5 layer based features showed highest identification rate in case of AlexNet-freez-MNIST up to $94.97 \%$ (96.34\% precision) and then pass the learned features to a linear SVM model for classification and identification of PD and $\mathrm{H}$ instance. The finetuneMNIST features based networks showed less accuracy as compare to other networks. In proposed study, ImageNet finetuned based approach outperformed and achieved $98.28 \%$ accuracy as compare to the accuracy $(96.69 \%$ accuracy $(97.21 \%$ precision) based on the fusion of fc6 and fc7 using AlexNet-freeze-ImageNet features on spiral drawing patterns.

Our proposed system showed the promising results on PaHaW (spiral data) using different approaches of deep transfer learning and of data-augmentation based techniques as compare to the existing studies in the literature.

\section{Conclusion}

Parkinson's Disease (PD) is hot neurological problem nowadays and the diagnosis of PD at early stages can cure PD and saves patient's life. In this study, we cope with the problem of Parkinson's disease identification through deep learner. For this purpose, we 
TABLE 11: Performance comparison of Parkinson's disease detection system

\begin{tabular}{|l|l|l|l|l|}
\hline Study & Features & Classifier & Dataset & Accuracy (\%) \\
\hline Pereira et al. [35] & Pen base features & CNN & HandPD & 80.19 \\
\hline Pereira et al. [36] & Pen base features & CNN & HandPD & 90.38 \\
\hline Pereira et al. [37] & CNN based features & CNN & HandPD & 93.50 \\
\hline Moetesum et al. [40] & $\begin{array}{l}\text { AlexNet-freeze-ImageNet } \\
\text { features from fc7 layer }\end{array}$ & SVM & PaHaW & 83 \\
\hline Proposed System & $\begin{array}{l}\text { AlexNet-freeze-MNIST fea- } \\
\text { tures fron conv5 layer }\end{array}$ & SVM & PaHaW & 94.97 \\
\hline Proposed System & $\begin{array}{l}\text { AlexNet-freeze-ImageNet } \\
\text { features from fc6+fc7 layers }\end{array}$ & SVM & PaHaW & 96.69 \\
\hline Proposed System & $\begin{array}{l}\text { Fine-tuned-ImageNet } \\
\text { features }\end{array}$ & AlexNet & PaHaW & 98.28 \\
\hline
\end{tabular}

considered the non-invasive method using handwriting images for detection of PD. The AlexNet classifier with transfer learning technique is proposed for detection of handwriting impairments in Parkinson's patients so as improving the diagnosis of PD. Basically, the idea is to model the handwriting features from AlexNet and transfer these features to our target data due to finite number of samples. So for, we have used the pre-trained Alexnet-ImageNet and AlexNetMNIST. We explored the two common approaches (Freeze and fine-tuning) for transfer learning using two different source datasets (MNIST and ImageNet). The Fine-tuned approach of transfer learning using ImageNet proved to be an efficient feature extraction approach and outperformed the state of the art systems. Without extraction of traditional features, CNN system achieved accuracy of $98.28 \%$ on spiral pattern. The advantages of the proposed deep learner classifier summarized as follows. The proposed classifier has the capacity to automatically extract hidden features, which extensively increases the performance of the classifier. Parkinsons disease can be diagnosed remotely and monitored using the proposed classifier. Therefore, patients rarely need to make visits to the clinic. The deep learner classifier can be used as a reliable classifier for the PD, results show that the proposed classifier outperforms the conventional methods. From proposed study, we concluded that spiral images for Parkinson's detection is more informative as compared to letters, words and sentences. As PaHaW dataset contains less spiral pattern that we increases by data augmentation but collection of huge amount of spiral dataset consider more efficient for Parkinson's detection.

In regard to future work, we aim to extend our study to other deep learner models (Google-net, resnet and VGG) and compare their performance on Pa$\mathrm{HaW}$ dataset, HandPD dataset or combination drawing of both. We also extend our work to other dataset of Parkinson's disease i.e. voice and images dataset for effective diagnosis of Parkinson's.

\section{Conflict of Interest}

The authors whose names are listed in this paper certify that they have NO conflict of interest.

\section{References}

[1] D. Standaert, M. Saint-Hilaire, and C. thomas, Parkinsons Disease Handbook. American Parkinson Disease Association, 2015. [Online]. Available: https://www.apdaparkinson.org/ wp-content/uploads/2017/02/APDA1703_ Basic-Handbook-D5V4-4web.pdf

[2] L. C. Grandi, G. Di Giovanni, and S. Galati, "Animal models of early-stage parkinsons disease and acute dopamine deficiency to study compensatory neurodegenerative mechanisms," Journal of neuroscience methods, 2018.

[3] E. Masliah, E. Rockenstein, I. Veinbergs, M. Mallory, M. Hashimoto, A. Takeda, Y. Sagara, A. Sisk, and L. Mucke, "Dopaminergic loss and inclusion body formation in $\alpha$-synuclein mice: implications for neurodegenerative disorders," Science, vol. 287, no. 5456, pp. 1265-1269, 2000.

[4] A. Letanneux, J. Danna, J.-L. Velay, F. Viallet, and S. Pinto, "From micrographia to parkinson's disease dysgraphia," Movement Disorders, vol. 29, no. 12, pp. 1467-1475, 2014.

[5] M. Thomas, A. Lenka, and P. Kumar Pal, "Handwriting analysis in parkinson's disease: Current status and future directions," Movement Disorders Clinical Practice, vol. 4, no. 6, pp. 806-818, 2017.

[6] Y. Crespo, M. Soriano, S. Iglesias-Parro, J. Aznarte, and A. Ibáñez-Molina, "Spatial analysis of handwritten texts as a marker of cognitive control," Journal of motor behavior, pp. 1-10, 2017.

[7] J. Collett, M. Franssen, C. Winward, H. Izadi, A. Meaney, W. Mahmoud, M. Bogdanovic, M. Tims, D. Wade, and H. Dawes, "A long-term self-managed handwriting intervention for people with parkinsons disease: results from the control group of a phase ii randomized controlled trial," Clinical rehabilitation, vol. 31, no. 12, pp. 1636-1645, 2017. 
[8] E. Nackaerts, S. Broeder, M. P. Pereira, S. P. Swinnen, W. Vandenberghe, A. Nieuwboer, and E. Heremans, "Handwriting training in parkinsons disease: A trade-off between size, speed and fluency," PloS one, vol. 12, no. 12, p. e0190223, 2017.

[9] J. C. Vasquez-Correa, J. R. Orozco-Arroyave, R. Arora, E. Nöth, N. Dehak, H. Christensen, F. Rudzicz, T. Bocklet, M. Cernak, H. Chinaei et al., "Multi-view representation learning via gcca for multimodal analysis of parkinson's disease," in Acoustics, Speech and Signal Processing (ICASSP), 2017 IEEE International Conference on. IEEE, 2017, pp. 2966-2970.

[10] M. Moetesum, I. Siddiqi, N. Vincent, and F. Cloppet, "Assessing visual attributes of handwriting for prediction of neurological disordersa case study on parkinsons disease," Pattern Recognition Letters, 2018.

[11] L. di Biase, S. Summa, J. Tosi, F. Taffoni, M. Marano, A. Cascio Rizzo, F. Vecchio, D. Formica, V. Di Lazzaro, G. Di Pino et al., "Quantitative analysis of bradykinesia and rigidity in parkinsons disease," Frontiers in neurology, vol. 9, p. 121, 2018.

[12] P. Werner, S. Rosenblum, G. Bar-On, J. Heinik, and A. Korczyn, "Handwriting process variables discriminating mild alzheimer's disease and mild cognitive impairment," The Journals of Gerontology Series B: Psychological Sciences and Social Sciences, vol. 61, no. 4, pp. P228-P236, 2006.

[13] I. Razzak, M. Imran, and G. Xu, "Efficient brain tumor segmentation with multiscale twopathway-group conventional neural networks," IEEE journal of biomedical and health informatics, 2018.

[14] M. I. Razzak, S. Naz, and A. Zaib, "Deep learning for medical image processing: Overview, challenges and the future," in Classification in BioApps. Springer, 2018, pp. 323-350.

[15] M. I. Razzak and S. Naz, "Microscopic blood smear segmentation and classification using deep contour aware cnn and extreme machine learning," in Computer Vision and Pattern Recognition Workshops (CVPRW), 2017 IEEE Conference on. IEEE, 2017, pp. 801-807.

[16] S. Naz, A. I. Umar, R. Ahmad, I. Siddiqi, S. B. Ahmed, M. I. Razzak, and F. Shafiat, "Urdu Nastaliq recognition using convolutional recursive deep learning," NeuroComputing, vol. 243, pp. 80-87, 2017.

[17] S. Naz, A. I. Umar, R. Ahmad, S. B. Ahmed, S. H. Shirazi, and M. I. Razzak, "Urdu Nastaliq text recognition system based on multi-dimensional recurrent neural network and statistical features," Neural computing and applications, vol. 28, no. 2, pp. 219-231, 2017.

[18] S. Naz, A. I. Umar, R. Ahmad, S. B. Ahmed, I. Sid- diqi, and M. I. Razzak, “Offline cursive Nastaliq script recognition using multidimensional recurrent neural networks with statistical features," NeuroComputing, vol. 177, pp. 228-241, 2016.

[19] A. Rehman, S. Naz, M. I. Razzak, and I. A. Hameed, "Automatic visual features for writer identification: A deep learning approach," Neural computing and applications, 2019.

[20] J. McLennan, K. Nakano, H. Tyler, and R. Schwab, "Micrographia in parkinson's disease," Journal of the neurological sciences, vol. 15, no. 2, pp. 141-152, 1972.

[21] A. Tsanas, M. A. Little, P. E. McSharry, J. Spielman, and L. O. Ramig, "Novel speech signal processing algorithms for high-accuracy classification of parkinson's disease," IEEE transactions on biomedical engineering, vol. 59, no. 5, pp. 12641271, 2012.

[22] L. Millian-Morell, T. Lopez-Alburquerque, A. Rodriguez-Rodriguez, R. Gomez-Nieto, J. Carro, J. J. Meilan, F. Martinez-Sanchez, C. Sancho, and D. E. Lopez, "Relations between sensorimotor integration and speech disorders in parkinson's disease," Current Alzheimer Research, vol. 15, no. 2, pp. 149-156, 2018.

[23] M. Hariharan, K. Polat, and R. Sindhu, "A new hybrid intelligent system for accurate detection of parkinson's disease," Computer methods and programs in biomedicine, vol. 113, no. 3, pp. 904-913, 2014.

[24] J. Rusz, R. Čmejla, H. Ržičková, J. Klempíř, V. Majerová, J. Picmausová, J. Roth, and E. Ržička, "Acoustic assessment of voice and speech disorders in parkinson's disease through quick vocal test," Movement Disorders, vol. 26, no. 10, pp. 1951-1952, 2011.

[25] M. Pettorino, E. Pellegrino, and M. G. Busà, "Speech disorders and parkinson's disease," Parkinsonism \& Related Disorders, vol. 22, p. e48, 2016.

[26] S. Aich, K. Younga, K. L. Hui, A. A. Al-Absi, and M. Sain, "A nonlinear decision tree based classification approach to predict the parkinson's disease using different feature sets of voice data," in Advanced Communication Technology (ICACT), 2018 20th International Conference on. IEEE, 2018, pp. 638-642.

[27] A. Caliskan, H. Badem, A. Basturk, and M. E. Yuksel, "Diagnosis of the parkinson disease by using deep neural network classifier," Istanbul University-Journal of Electrical \& Electronics Engineering, vol. 17, no. 2, pp. 3311-3319, 2017.

[28] M. Delrobaei, S. Memar, M. Pieterman, T. W. Stratton, K. McIsaac, and M. Jog, "Towards remote monitoring of parkinsons disease tremor using wearable motion capture systems," Journal of the neurological sciences, vol. 384, pp. 38-45, 2018.

[29] J. Cancela, M. Pastorino, and M. T. A. Waldmeyer, 
"Trends and new advances on wearable and mobile technologies for parkinson's disease monitoring and assessment of motor symptoms: How new technologies can support parkinson's disease," in Biomedical Engineering: Concepts, Methodologies, Tools, and Applications. IGI Global, 2018, pp. 1180-1204.

[30] Y. Xia, Z. Yao, Y. Lu, D. Zhang, and N. Cheng, "A machine learning approach to detecting of freezing of gait in parkinson's disease patients," Journal of Medical Imaging and Health Informatics, vol. 8, no. 4, pp. 647-654, 2018.

[31] C. Xu, J. He, X. Zhang, C. Wang, and S. Duan, "Template-matching-based detection of freezing of gait using wearable sensors," Procedia Computer Science, vol. 129, pp. 21-27, 2018.

[32] B. M. Eskofier, S. I. Lee, J.-F. Daneault, F. N. Golabchi, G. Ferreira-Carvalho, G. Vergara-Diaz, S. Sapienza, G. Costante, J. Klucken, and T. Kautz, "Recent machine learning advancements in sensor-based mobility analysis: deep learning for parkinson's disease assessment," in IEEE 38th Annual International Conference of the Engineering in Medicine and Biology Society (EMBC). IEEE, 2016, pp. 655-658.

[33] V. Ruonala, E. Pekkonen, O. Airaksinen, M. Kankaanpää, P. A. Karjalainen, and S. M. Rissanen, "levodopa-induced changes in electromyographic patterns in patients with advanced parkinsons disease," Frontiers in neurology, vol. 9, p. 35, 2018.

[34] A. E. Bond, B. B. Shah, and W. J. Elias, "Assessing tremor and adverse events in patients with tremor-dominant parkinson disease undergoing focused ultrasound thalamotomyreply," JAMA neurology, vol. 75, no. 5, pp. 633-633, 2018.

[35] C. R. Pereira, S. A. Weber, C. Hook, G. H. Rosa, and J. P. Papa, "Deep learning-aided parkinson," in 2016 29th SIBGRAPI Conference on Graphics, Patterns and Images (SIBGRAPI). IEEE, 2016, pp. 340-346.

[36] C. R. Pereira, D. R. Pereira, J. P. Papa, G. H. Rosa, and X.-S. Yang, "Convolutional neural networks applied for parkinsons disease identification," in Machine Learning for Health Informatics. Springer, 2016, pp. 377-390.

[37] C. R. Pereira, D. R. Pereira, G. H. Rosa, V. H. Albuquerque, S. A. Weber, C. Hook, and J. P. Papa, "Handwritten dynamics assessment through convolutional neural networks: An application to parkinson's disease identification," Artificial intelligence in medicine, vol. 87, pp. 67-77, 2018.

[38] Y. Zhang, "Can a smartphone diagnose parkinson disease? a deep neural network method and telediagnosis system implementation," Parkinsons Disease, vol. 2017, 2017.

[39] S. Grover, S. Bhartia, A. Yadav, K. Seeja et al., "Predicting severity of parkinsons disease using deep learning," Procedia Computer Science, vol. 132, pp. 1788-1794, 2018.

[40] M. Moetesum, I. Siddiqi, N. Vincent, and F. Cloppet, "Assessing visual attributes of handwriting for prediction of neurological disordersa case study on parkinsons disease," Pattern Recognition Letters, 2018.

[41] H. Choi, S. Ha, H. J. Im, S. H. Paek, and D. S. Lee, "Refining diagnosis of parkinson's disease with deep learning-based interpretation of dopamine transporter imaging," NeuroImage: Clinical, vol. 16, pp. 586-594, 2017.

[42] L. C. Afonso, G. H. Rosa, C. R. Pereira, S. A. Weber, C. Hook, V. H. C. Albuquerque, and J. P. Papa, "A recurrence plot-based approach for parkinsons disease identification," Future Generation Computer Systems, 2018.

[43] D. Gupta, A. Julka, S. Jain, T. Aggarwal, A. Khanna, N. Arunkumar, and V. H. C. de Albuquerque, "Optimized cuttlefish algorithm for diagnosis of parkinsons disease," Cognitive Systems Research, 2018.

[44] D. Gupta, S. Sundaram, A. Khanna, A. E. Hassanien, and V. H. C. de Albuquerque, "Improved diagnosis of parkinson's disease using optimized crow search algorithm," Computers $\mathcal{E}$ Electrical Engineering, vol. 68, pp. 412-424, 2018.

[45] J. Ratliff, R. A. Ortega, H. Y. Ooi, A. Mirallave, A. Glickman, Q. Yu, D. Raymond, S. Bressman, S. Pullman, and R. Saunders-Pullman, "Digitized spiral analysis may be a potential biomarker for brachial dystonia," Parkinsonism $\mathcal{E}$ related disorders, 2018.

[46] M. San Luciano, C. Wang, R. A. Ortega, Q. Yu, S. Boschung, J. Soto-Valencia, S. B. Bressman, R. B. Lipton, S. Pullman, and R. Saunders-Pullman, "Digitized spiral drawing: A possible biomarker for early parkinsons disease," PloS one, vol. 11, no. 10, p. e0162799, 2016.

[47] R. Saunders-Pullman, C. Derby, K. Stanley, A. Floyd, S. Bressman, R. B. Lipton, A. Deligtisch, L. Severt, Q. Yu, M. Kurtis et al., "Validity of spiral analysis in early parkinson's disease," Movement disorders: official journal of the Movement Disorder Society, vol. 23, no. 4, pp. 531-537, 2008.

[48] N. Aly, J. Playfer, S. Smith, and D. Halliday, "A novel computer-based technique for the assessment of tremor in parkinson's disease," Age and Ageing, vol. 36, no. 4, pp. 395-399, 2007.

[49] P. Drotr, J. Mekyska, I. Rectorova, L. Masarova, Z. Smekal, and M. Faundez-Zanuy, "Analysis of in-air movement in handwriting: A novel marker for parkinsons disease," Computer Methods and Programs in Biomedicine, vol. 117, pp. 405-411, 2014.

[50] A. Krizhevsky, I. Sutskever, and G. E. Hinton, "Imagenet classification with deep convolutional neural networks," in Advances in neural information 
processing systems, 2012, pp. 1097-1105.

[51] P. H. Kraus and A. Hoffmann, "Spiralometry: computerized assessment of tremor amplitude on the basis of spiral drawing," Movement Disorders, vol. 25, no. 13, pp. 2164-2170, 2010.

[52] K. Stanley, J. Hagenah, N. Brüggemann, K. Reetz,
L. Severt, C. Klein, Q. Yu, C. Derby, S. Pullman, and R. Saunders-Pullman, "Digitized spiral analysis is a promising early motor marker for parkinson disease," Parkinsonism $\mathcal{E}$ related disorders, vol. 16, no. 3, pp. 233-234, 2010. 